\title{
Modeling the cyclic modulation of photospheric lines
}

\author{
V. Penza ${ }^{1,2}$, E. Pietropaolo ${ }^{3}$, and W. Livingston ${ }^{4}$ \\ 1 INAF - Osservatorio Astronomico di Roma, via Frascati 33, 00040 Monte Porzio Catone, Italy \\ e-mail: penza@roma2.infn.it \\ 2 Universitá di Roma "Tor Vergata”, via della Ricerca Scientifica 1, 00133 Roma, Italy \\ 3 Dipartimento di Fisica, Universitá degli Studi di L'Aquila, via Vetoio, 67010 Coppito, L'Aquila, Italy \\ ${ }^{4}$ National Solar Observatory, PO Box 26732, Tucson, AZ 85726, USA
}

Received 11 May 2005 / Accepted 9 March 2006

ABSTRACT

\begin{abstract}
We have studied the behavior of three photospheric lines (Fe I 537.9, C I 538.0 and Ti II $538.1 \mathrm{~nm}$ ), which have been monitored on the Sun for more than twenty years, either as full-disk or as center-disk measurements (Gray \& Livingston 1997; Livingston \& Wallace 2003). The aim is to detect a possible photospheric variation with the cycle.

We try to reconstruct the cyclic variations of full-disk line depths as due to active region (AR) modulation through a spectral synthesis with FAL semi-empirical models (Fontenla et al. 1999) weighted by AR coverage factors. The sensitivity of these lines to thermodynamic variations and to AR presence is analyzed.

We show that the AR modulation alone cannot explain all the observational results, either in amplitude or in phase. The "residual", i.e. the difference between observed behavior of these three lines at full-disk and that predicted by models for the AR modulation, results in a signal that is correlated with the measured center-disk line variations, and should be free from magnetic effects.

Both the full-disk and the center-disk data show several periodicities; furthermore there are two periodicities shared by the three lines, one close to the $11 \mathrm{yr}$ magnetic cycle and the other of $2.8 \mathrm{yr}$.
\end{abstract}

Key words. Sun: activity - Sun: faculae, plages

\section{Introduction}

Most solar indices such as sunspot number, 10-cm flux, $\mathrm{Ca} \mathrm{K}$ index, etc. represent, in one way or another, the presence of surface magnetism. The total solar irradiance (TSI) and the strengths of weak photospheric lines are particularly interesting. The TSI is affected by sunspot blocking and bright faculae, and by any large scale photospheric temperature change. The weak photospheric lines observed on the full disk, however, are only slightly affected by faculae, and not at all by sunspot blocking; center disk magnetic features are negligible in area and they have no effect on the TSI. The question arises: can photospheric lines then be used to discriminate between different mechanisms that determine solar irradiance variations?

The TSI (i.e. the energetic flux emitted by the Sun, integrated over the whole wavelength range as observed from space, and measured at $1 \mathrm{AU}$ ) varies about $0.10 \%$ in phase with magnetic activity (Fröhlich 1994). Superposed on this 11-year trend there are variations on shorter times scales (hours and days) that are found to be wholly due to the presence of active regions (ARs) (Sofia et al. 1982; Chapman et al. 1986). Spots, faculae and enhanced network are the cause of a large part of the observed variations over magnetic cycle time-scales (Willson \& Hudson 1991; Krivova et al. 2003), but the existence of a variation in the quiet photosphere component is still debated (Lydon et al. 1996; Penza et al. 2003). In other words, in the literature there are two views: the first one ascribes all irradiance variations to the evolution of surface magnetic fields and that these features are superposed to an otherwise constant background; the second one says that the solar global structure may change as a consequence of variations of the magnetic field both in the solar interior, where the dynamo process operates, and on the surface, where activity modifies the boundary conditions.

There are indications of the existence of a fine structure in solar activity cycles. The literature contains examples of the quasibiennial variations, better known as quasi-biennial oscillations (QBOs, see e.g. Gnevyshev 1977; Benevolenskaya 1998; Kane 2005). Similar variations within the same time interval are found in many geophysical processes (see e.g. Baldwin et al. 2001; McIntyre 1994).

The study of photospheric lines could represent a method to detect global changes of the Sun not directly linked to the presence of active regions, even if their sensitivity to different parameters and to AR presence have to be taken into account (Caccin et al. 2002; Penza et al. 2004b). Since 1978 three photospheric lines (Fe I $537.958 \mathrm{~nm}$, C I $538.032 \mathrm{~nm}$ and Ti II $538.103 \mathrm{~nm}$ ) have been monitored on the Sun and their line depths and equivalent widths measured, both full-disk (Gray \& Livingston 1997a) and at center-disk (Livingston \& Wallace 2003). The characteristics of these lines, their response to any temperature changes, and the active region effects on them, have been analyzed in a previous paper (Penza et al. 2004b).

In this work we try to account for the cyclic behavior of spectral lines by implementing a simplified model in order to reconstruct line depth trends along the solar cycle. We estimate how much of the line-depth variations measured at full disk can be attributed to active region modulation, and show that a part of it must be derived from other phenomena.

Our hypothesis is that the signal of this "residual", i.e. the difference between the full-disk measurements and the AR contribution, can be found in the line depth measurements at center disk. 

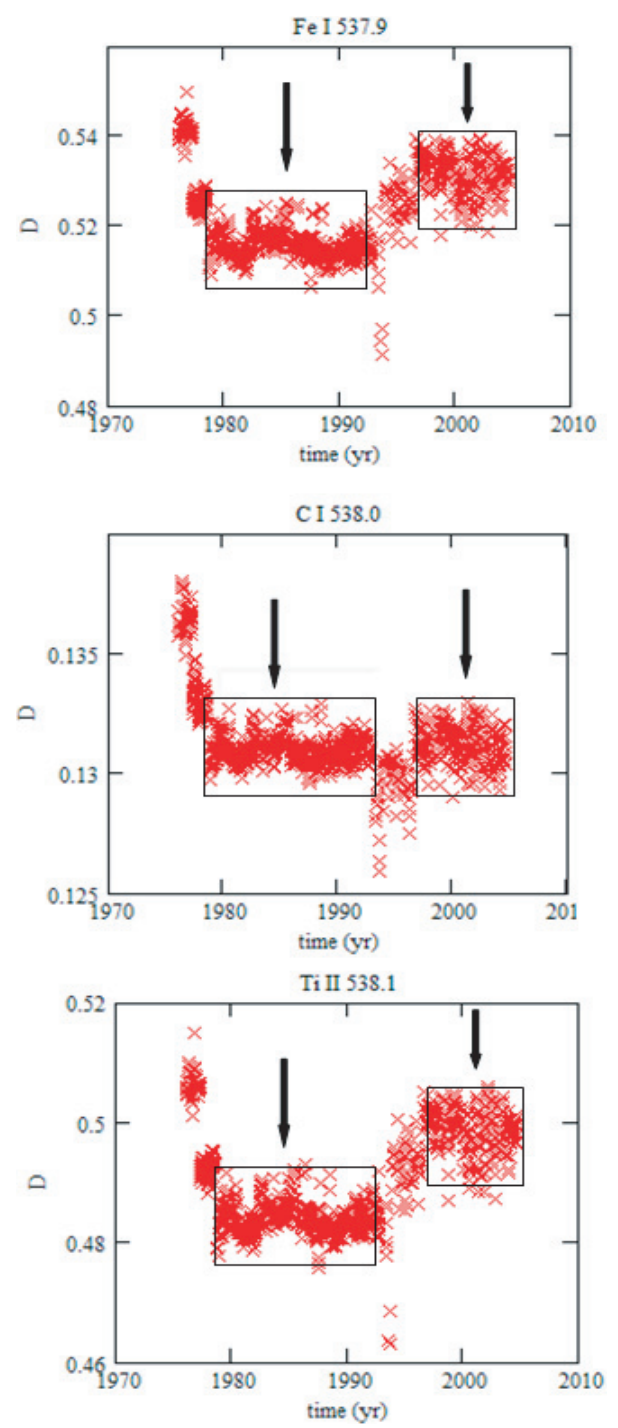

Fig. 1. Absolute depths of the three lines measured since 1976. The data actually used for the comparison with the reconstruction are indicated in the boxes by vertical arrows.

We also note the existence of a shared periodicity (for all of the line data and for other solar activity indicators) of about $2-3$ years.

\section{Observations}

All data used in this study were taken with the Kitt Peak 13.5-m scanning spectrometer operating in double pass.

Figure 1 shows the over 25 years of collected raw data from this program. The initial changes in line depth from 1975 to 1977 were a result of experimentation with optical filters and slit widths. After 1978 these components were fixed, and the system was immutable until a new and larger grating was installed in 1992.

For "Sun-as-a-star", or "full-disk", measurements unfocussed sunlight is delivered to the spectrograph where a $0.5 \times$ $10.0 \mathrm{~mm}$ entrance slit causes a pinhole image of the Sun to be formed on the grating. In the 5th order, where we work, the projected grating size was, until 1992, about $15 \times 15 \mathrm{~cm}$. There are two negative consequences of this method of grating illumination: first, there is a sensitivity to day-to-day telescope alignment plus the annular variation in the apparent solar diameter, and second, there is a potentially reduced spectral resolution. An analysis of this data set (Gray \& Livingston 1997a) has detected the annular variation of the solar diameter arising from the Earth's elliptical orbit as the image varies on the grating.

Spectral resolution is a function of the number of grating grooves covered by coherent light, and, of course, the entrance and exit slit widths. Imagine a perfectly sharp image of the Sun on the grating. No spectrum would result because there would be no coherence between the rulings! In practice, diffraction at the $0.5 \mathrm{~mm}$ entrance slit produces about $2 \mathrm{~cm}$ of coherent light, far less than a grating completely covered with coherent light. This reduction in resolution is, however, not very important. Resolution is set mainly by our relatively wide slit widths. A ray tracing program informs us that the resolution is about 106000 . In the fall of 1992 the grating size was increased and again there was a period of experimentation that is evident in Fig. 1. Beginning in 1996 the system was again fixed, but now there were two gratings (visible and infrared). Different observers re-aligned the system, the grating was somewhat nonuniform over its surface, and all this led to the increased variance seen from 1996 to the present. Also, because the new larger grating sampled the limb more fully, we were in effect observing a slightly different Sun.

To avoid all the above effects, this paper's full disk data analysis is confined to 1978.8-1992.7, with 1996.6-2004.5 treated separately, as shown in Fig. 1.

Besides full-disk, there were also "center-disk" observations (Livingston \& Wallace 2003). Except for the "experimental epochs" of 1976-78 and 1993-96, the center-disk data are free of the above full disk problems; the entire grating is illuminated by coherent light, and because the old and new gratings had the same ruling pitch, the resolution is constant with time. For a center-disk observation, a small lens is placed in the beam in front of the spectrograph in a way that the $80 \mathrm{~cm}$ image is optically averaged over about 2 arcmin. The aim is to avoid disk fine structure. Additionally, using chromospheric Ca K intensity as a guide, the image may be positioned somewhat off center to avoid any activity. Thus, center-disk archives represent the quiet sun.

An observation consists of 30 scans which are co-added at the telescope. Using the data reduction program DECOMP, developed by J. Brault at Kitt Peak, each resulting record is normalized to a three point continuum and line intensities and equivalent widths (over $120 \mathrm{m \AA}$ ) are extracted. Both Fe 537.9 and C $538.0 \mathrm{~nm}$ are blended with weak water vapor lines. From observations of telluric water at $1083.21 \mathrm{~nm}$ precipitable water in cc for one air mass is determined for each day. An empirical correction is then applied to the spectra to remove water. Such water corrections appreciably reduce variance in both full disk and center disk time series.

\section{The line responses}

\subsection{Thermodynamical response}

We analyze the response of the lines referred to above to thermodynamic variations in order to recognize which thermodynamic effects cause the lines to become deeper or shallower.

The sensitivity of these lines to the temperature variations was already analyzed by Caccin et al. (2002); in particular, the authors studied the calibration between the variations of the line depth ratios and those of $T_{\text {eff }}$, showing that this approach could not be used in order to infer the $T_{\text {eff }}$ solar variations. An incompatibility between the observed behavior of individual lines and 

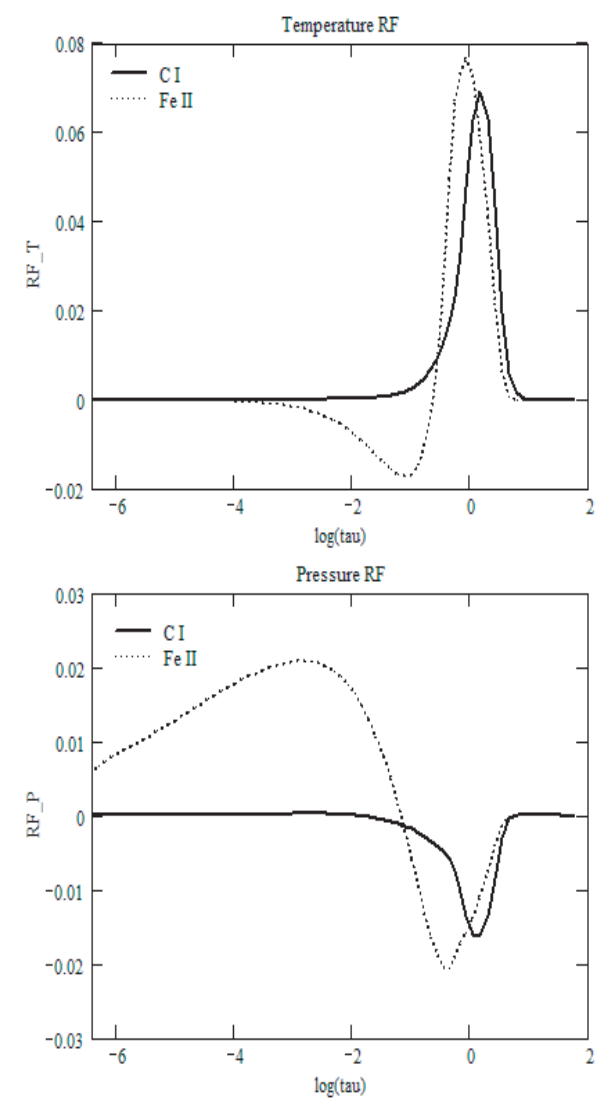

Fig. 2. Temperature (up) and pressure (down) Response Functions for the depth of Fe I and C I. The $R F \mathrm{~s}$ are arbitrarily rescaled in order to represent the functions in a unique plot.

the opposite sensitivity to the temperature line variations was noted by Gray \& Livingston (1997a). In fact, the observed line depths vary concurrently (Fig. 5 of Gray \& Livingston 1997a), contrary to the theoretical prediction that the $\mathrm{C}$ line should deepen with increasing $T_{\text {eff }}$, the Fe line become shallower and the Ti line should not vary. The authors initially ascribed this trend to some multiplicative instrumental effect, e.g. scattered light, and got over the problem by using not the individual lines, but their ratios $(\mathrm{C} / \mathrm{Fe}$ and $\mathrm{C} / \mathrm{Ti})$. These ratios should be independent of the amount of scattered light. Afterwards the same authors excluded this instrumental effect as the cause of the line behavior, because there is not a unique correction factor for all of the three lines, and scattered light is compensated by the double pass system (Gray \& Livingston 1997b).

The parallel trend of the lines could have other explanations, because temperature is not the only thermodynamic quantity which can vary to cause the $\mathrm{C}$ and Fe lines to have an opposite behavior. This is clear by studying the Response Function $(R F$, Caccin et al. 1977) of their depths, partly analyzed by Penza et al. (2004b). In Fig. 2 we report the temperature and pressure line depth $R F\left(R F_{\mathrm{D}}\right)$ of the Fe I and $\mathrm{C} \mathrm{I}$ lines, which is given by:

$R F_{\mathrm{D}}=-\left(R F_{\lambda}-R F_{\mathrm{c}}\right)$

where $R F_{\mathrm{c}}$ and $R F_{\lambda}$ are the continuum and the line center $R F$, respectively.

We note that the $\mathrm{C}$ depth has a positive temperature $R F$ everywhere, so any positive temperature perturbation $(\delta T>0)$ generates a positive variation of the line depth $(D)$, this being

$\delta D=\int_{0}^{\infty} R F(\tau) \delta T(\tau) \mathrm{d} \tau$
The Fe I case is less obvious, because its $R F$ does not present an unique sign. Its behavior depends on the kind of temperature perturbation and on its structure in the atmosphere layers. If we consider as $\delta T$ the difference between two temperature structures corresponding to two stars having different $T_{\text {eff }}$, then $\delta T>0$ leads to $\delta D<0$, but we can have a different behavior if, e.g., the perturbation is localized only around $\tau=0$. The situation is similar when we take into account the pressure $R F \mathrm{~s}$.

As noted in Caccin et al. (2002), the assumption that the changes occurring in the Sun during its magnetic cycle are correctly simulated by using the modifications of the atmospheric stratification with $T_{\text {eff }}$ along the main sequence could be wrong. Moreover, other phenomena should be taken into account, like multidimensional effects on line formation due to variable granulation or a simple change of the mean velocity field. In fact, if the mean velocity field increases, all the lines respond in the same way, i.e. by becoming broader and shallower. The effect of a granulation variation (that has not yet been confirmed) is more complicated to define. Caccin \& Penza (2000) developed a simple analytic model of the granulation and analyzed the possible effects of granule dimension variation on these lines; the authors showed that the line depths of all the lines increase (not linearly) with the granule dimension (e.g. for a variation of about $30 \%$ of the mean granule dimension $\delta D / D$ is about $0.004 \%$ for the Fe I and $0.008 \%$ for the C I).

\subsection{AR model responses}

The response of the line depths to atmospheric models corresponding to different solar regions, i.e. the sensitivity of the three lines to the presence of bright active regions, was analyzed in Penza et al. (2004b). Here we report only the pertinent results.

A spectral synthesis was computed via the SPECTRUM program by R. O. Gray (1994) in LTE, and the FAL semiempirical models (Fontenla et al. 1999), in order to reproduce the atmosphere in the active regions. The characteristics of the atmosphere models that we used and their capability to reproduce experimental data have been described in a previous paper (Penza et al. 2004a) where the authors analyzed which of the FAL models were able to reproduce the average continuum AR contrast.

We used modified models (Unruh et al. 1999; Penza et al. 2004a), where the temperature structure is truncated around the temperature minimum and then extrapolated down to lower temperatures, with a trend similar to that of Kurucz's models (Kurucz 1994).

Penza et al. (2004a) showed that it is not possible to define a unique model for the faculas or the network. One can, however, find an average model that reproduces the center-to-limb variations of the structure contrasts. In Table 1, we report the results of the line depths of Fe I, C I and Ti II in the models of interest: modC, modE, modF, modH and modP, representing average sun, average network, faint plage, average plage and bright plage, respectively.

The comparison with observations previously made by Penza et al. (2004b) suggests that the best models to reproduce the observed behavior of Fe I and Ti II lines in the facular zone were modF and modH. A similar result came from the analysis of the continuum contrasts (Penza et al. 2004a).

The behavior of C I at disk center proved more complex. Nevertheless, we use the same models in the calculation of the active region contribution for all the lines.

For the sunspot, we choose a model that simulates a mean of umbra and penumbra, like a Kurucz model with $T_{\text {eff }}=5150 \mathrm{~K}$, as suggested by Unruh et al. (2000). 
Table 1. The line depths and adjacent continuum flux for FAL models. The continuum flux unit is $10^{6} \mathrm{erg} \mathrm{s}^{-1} \mathrm{~cm}^{-2}$.

\begin{tabular}{lllll}
\hline \hline Model & $D(\mathrm{Fe})$ & $D(\mathrm{C})$ & $D(\mathrm{Ti})$ & $I_{\mathrm{c}}$ \\
\hline ModC & 0.632 & 0.142 & 0.600 & 3.126 \\
ModE & 0.609 & 0.144 & 0.589 & 3.135 \\
ModF & 0.581 & 0.147 & 0.575 & 3.155 \\
ModH & 0.528 & 0.148 & 0.549 & 3.264 \\
ModP & 0.473 & 0.144 & 0.521 & 3.349 \\
\hline
\end{tabular}

\section{Reconstruction of the line variations}

We reproduce the trend of the line depth (integrated over the disk) by weighting different model results with corresponding coverage factors, in analogy with TSI reconstructions in the literature (Fligge et al. 2000; Penza et al. 2003; Krivova et al. 2003).

This is given by:

$D=1-\frac{\sum_{j} \alpha_{j} I_{\lambda}^{j}}{\sum_{j} \alpha_{j} I_{\mathrm{c}}^{j}}=\frac{\sum_{j} \alpha_{j} I_{\mathrm{c}}^{j} D_{j}}{\sum_{j} \alpha_{j} I_{\mathrm{c}}^{j}}$,

where $\alpha_{j}$ is the coverage factor corresponding to the $j$ th structure (quiet sun, network, facula or spot), $I_{\lambda}^{j}$ is the corresponding intensity at the line center, and $I_{\mathrm{c}}^{j}$ the corresponding continuum.

In Eq. (3) we have supposed that the coverage factors are independent of disk position and we have used directly the fluxes (integrated over the disk), without considering the intensity of each structure at the heliocentric angle where it is located. Because we are interested in a yearly average, we retain this approximation as sensible. In the Appendix an estimate of the error in neglecting the $\mu$-dependence is reported. that

Through simple algebraic manipulations, and by imposing

$\alpha_{q}=1-\sum_{j \neq q} \alpha_{j}$

where the index $q$ corresponds to quiet Sun, we can write $D$ as

$D=D_{q} \frac{\sum_{j \neq q} \alpha_{j}\left(i_{\mathrm{c}}^{j} d_{j}-1\right)+1}{\sum_{j \neq q} \alpha_{j}\left(i_{\mathrm{c}}^{j}-1\right)+1}$

where $i_{\mathrm{c}}^{j}$ is the ratio between the continuum in the active region and the quiet $\operatorname{Sun}\left(i_{\mathrm{c}}^{j}=I_{\mathrm{c}}^{j \neq q} / I_{\mathrm{c}}^{q}\right)$, while $d_{j}$ is the analogous ratio between the line depths $\left(d_{j}=D_{j \neq q} / D_{q}\right)$. If we consider the denominator, we note that there are three terms in the sum: $\alpha_{\mathrm{n}}\left(i_{\mathrm{c}}^{\mathrm{n}}-1\right), \alpha_{\mathrm{f}}\left(i_{\mathrm{c}}^{\mathrm{f}}-1\right)$ and $\alpha_{\mathrm{s}}\left(i_{\mathrm{c}}^{\mathrm{s}}-1\right)$, where $\mathrm{n}, \mathrm{f}$ and $\mathrm{s}$ are network, faculae and spots, respectively. In Table 2 we estimate these terms, by using for $i_{\mathrm{c}}$ the values of Table 1 and the coverage data as evaluated in the next paragraph. For this estimate, because we want to maximize the values, we consider the mean of modE and modF intensity for the network and the mean of $\operatorname{modF}, \operatorname{modH}$ and modP for the facula. Finally, for the spot, we use a weighted mean of the $i_{\mathrm{c}}$ in the umbra and the penumbra. By looking at the table, we can note that all $\alpha\left\|i_{\mathrm{c}}-1\right\|$ terms are small with respect to 1 . Then we can do an expansion in Taylor series, stopping at the first order (i.e. neglecting the mixed term having the double summation), and we obtain

$D \cong D_{q}\left(\sum_{j \neq q} \alpha_{j} i_{\mathrm{c}}^{j}\left(d_{j}-1\right)+1\right)$.
Table 2. Maximum value of the terms of the summation in Eq. (5), where $\mathrm{n}, \mathrm{f}$ and $\mathrm{s}$ indicate network, facula and spot, respectively.

\begin{tabular}{l|lll}
\hline \hline Term & $\mathrm{n}$ & $\mathrm{f}$ & $\mathrm{s}$ \\
\hline$\alpha$ & 0.4 & 0.08 & 0.005 \\
$\left\|i_{\mathrm{c}}-1\right\|$ & 0.006 & 0.04 & 0.5 \\
\hline$\alpha\left\|i_{\mathrm{c}}-1\right\|$ & 0.0024 & 0.003 & 0.0025 \\
\hline
\end{tabular}

If we consider a perturbation of $D$, and take into account both the variation of $\alpha_{j}$ and a possible modification of $D_{q}$, we have

$\frac{\delta D}{D} \cong \frac{\delta D_{q}}{D_{q}}+\frac{\sum_{j \neq q} \delta \alpha_{j} i_{\mathrm{c}}^{j}\left(d_{j}-1\right)}{\sum_{j \neq q} \alpha_{j} i_{\mathrm{c}}^{j}\left(d_{j}-1\right)+1} \equiv \frac{\delta D_{q}}{D_{q}}+\frac{\delta D_{\mathrm{AR}}}{D_{\mathrm{AR}}}$

In other words, to first order, the variations of the background (quiet Sun) and those of the ARs are separated.

As a guarantee for the sufficiency of the first order in the perturbation of the second term in Eqs. (6) and (7) we compare the variations of $D$ provided by using directly Eq. (3) and those provided by $\delta D_{\mathrm{AR}}$ as in Eq. (7). We find that the error is $\leq 5 \times 10^{-5}$.

\subsection{Magnetic contribution}

Here we study the line variation as due to active region modulation alone. We consider in Eq. (7) only the term corresponding to AR variations, and we repeat the Taylor expansion of the denominator. In this case, a single term remains:

$\frac{\delta D_{\mathrm{AR}}}{D_{\mathrm{AR}}} \cong \sum_{j \neq q} \delta \alpha_{j} i_{\mathrm{c}}^{j}\left(d_{j}-1\right)$.

Regrettably, we do not have the data on AR area for all of Gray \& Livingston data years (1978-1992), but we can exploit the knowledge derived from Cycle 22 and 23 (1986-2002) and monitored by Chapman and Walton (private communication, for more details about this see e.g. Walton et al. $2003 a, 2003 b)$ in order to establish a relation between measured activity indexes and AR coverage, and then extend this relation to the years not covered by direct observations (1978-1985). As a proxy-index we use the Mg II index ( $\Xi_{\mathrm{Mg}}$, from www-iup.physik.uni-bremen/gome/solar) which is related to facular and spot area (with a linear correlation coefficient equal to 0.98 ) by:

$A_{\text {fac }}=3.583 \Xi_{\mathrm{Mg}}-0.464$

$A_{\text {spot }}=0.149 \Xi_{\mathrm{Mg}}-0.018$.

We note that these relations are very similar to those found by Penza et al. (2004c) by using the data of the PSPT telescope in Rome (Ermolli et al. 2001), relative to Cycle 23, so we also assume the estimate for the network coverage as derived from PSPT data:

$A_{\text {net }}=4.216 \Xi_{\mathrm{Mg}}-0.237$.

These estimates can be used to reconstruct the total solar irradiance variation as:

$\delta F=\sum_{j \neq q} \delta \alpha_{j}\left(F_{j}-F_{q}\right)$

We use modE and modF as network and facula models, respectively, and a mean contrast between an average spot (umbra and penumbra) and the quiet Sun as equal to 0.56. The latter is the estimate for an average zone of umbra (u) and penumbra (p); 


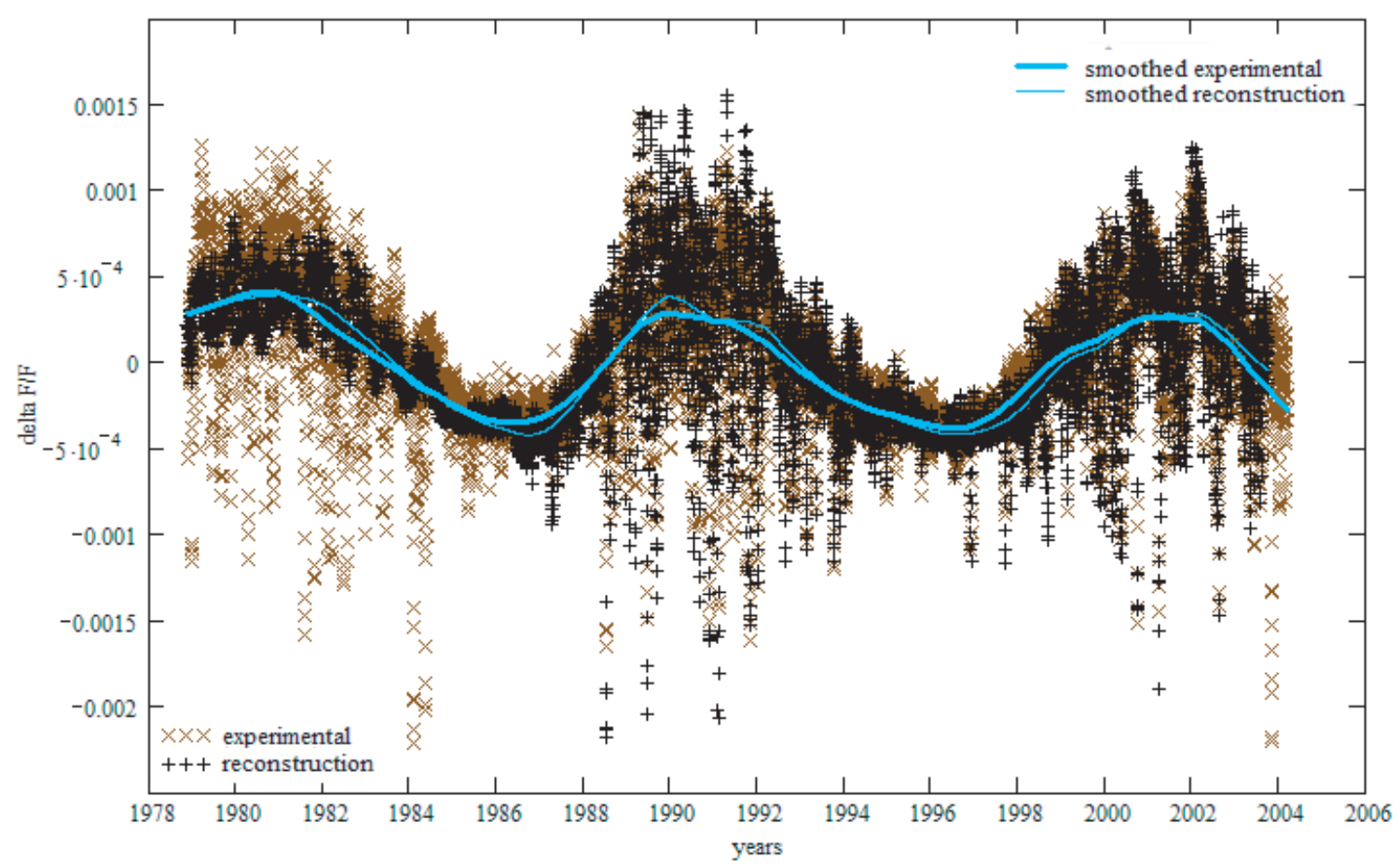

Fig. 3. Comparison between the observed TSI from VIRGO/SOHO (grey points) and the reconstruction obtained through models and spectral synthesis (black points).

we weight the corresponding contrasts $\left(F_{\mathrm{u}} / F_{q}=0.35\right.$ and $\left.F_{\mathrm{p}} / F_{q}=0.76\right)$, obtained by averaging the values found by Tritschler \& Schmidt $(2002)^{1}$, with their coverage ratio $(r=$ $\alpha_{\mathrm{p}} / \alpha_{\mathrm{u}}=3.6$ ), using Rome PSPT data (Penza 2003), i.e.

$\alpha_{\text {spot }} F_{\text {spot }}=\frac{\alpha_{\text {spot }}}{r+1} F_{\text {umbra }}+\alpha_{\text {spot }} \frac{r}{r+1} F_{\text {penumbra }}$.

The comparison between the reconstruction via models and the observed data from VIRGO/SOHO satellite (Fröhlich 2003, version 5-007-0310a) is shown in Fig. 3. The average TSI variations are well reproduced in the period where we have used the coverage factors obtained by calibration with the $\mathrm{Mg}$ index (1978-1985), while in the period where the direct estimate of the values of facular and spot area (1986-2003) are used, we are able to reproduce also the variations on shorter time-scales.

In Eq. (10) we have not considered possible variations of the quiet Sun, but this comparison has the only aim of showing that, with the coverage factors so estimated, and by considering modE and modF as good models for estimating network and facular contribution, we can accurately reproduce the TSI variations. We might then expect that an analogous reconstruction would work for line behavior. However, this is not the case. If we try to reconstruct the trend of line depths along the cycle, the result does not match very well with the observed data.

In Fig. 4 we show the reconstruction of the three line depth variations, using the same models as the TSI reconstruction. It is evident that there are problems both with respect to the amplitude and to the phase of the variations.

We have to note that the layers forming the $C$ line are very deep, and here the validity of the models is less guaranteed (Penza et al. 2004a; Fontenla et al. 2004). Even if a modification of the model is made, the variations derived from active regions

1 The results of Tritschler and Schmidt were obtained for wavelengths of $402.1,569.5$ and $709.1 \mathrm{~nm}$. in deep layers always remain too small to explain the observed variations.

In other words, if the observations are correct, this kind of reconstruction, that uses one-dimensional plane-parallel models of active regions, cannot reproduce all the phenomena affecting the spectral lines along the cycle. If we compare directly the variations of the line depth and those of the coverage of faculae (Fig. 6) we note that there is no obvious correspondence between the variability of the line depth and facular areas.

\subsection{The non-magnetic contribution}

Equation (7) shows that, to first order, the differences between the overall variations and those due to active regions should give an estimate of the (full-disk) background variations. Then, as a first (and rough) comparison, we can compare the difference between full-disk variations (observational data) and the modulation due solely to active regions (semi-empirical reconstruction) with the observational data (Livingston 2003) corresponding to variations of line depth at the center disk (Fig. 5). Actually, the two series of the values are not really comparable, since the fulldisk data could be affected also by a possible limb-darkening variation of the continuum and/or of the line depths. Moreover, the center-disk data are averaged over the granulation structure, which might change (Roudier 2003). Nevertheless, we suggest that these effects too, if they are present, would have the same origin, i.e. the same periodicity. Figure 5 shows just two trends that are similar in periodicity, although not much in amplitude. The correlation coefficients are $r=\simeq 0.7$ for Fe I and Ti II and $r=\simeq 0.4$ for C I. Actually, the center-disk data are also correlated with full-disk ones, with very similar correlation coefficients. Thus, it would seem that the phenomenon correlating the two data series (full and center-disk) is not linked to AR modulation, because this correlation is present even when the AR effect is removed. 

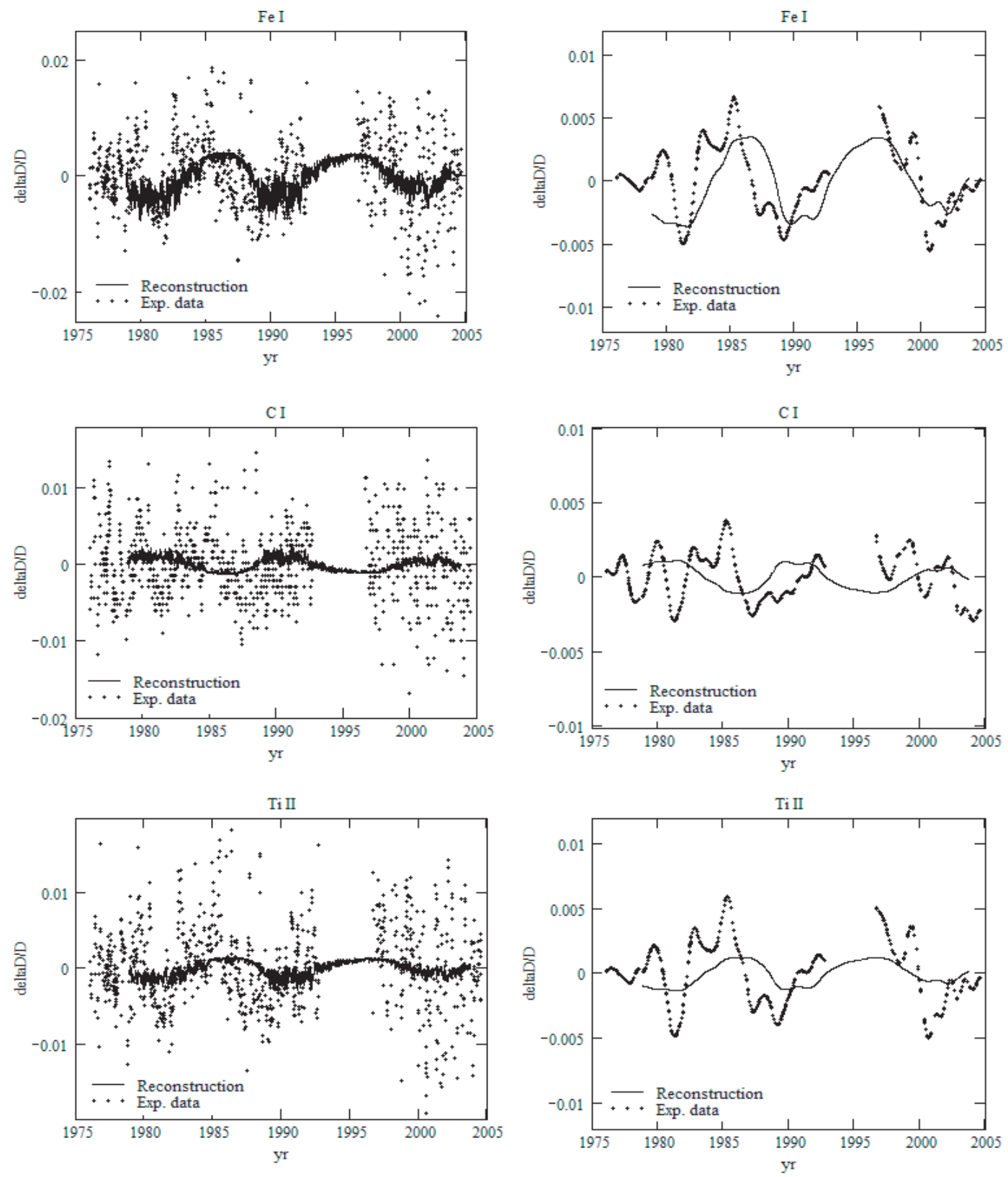

Fig. 4. Reconstruction of the line variations due to ARs alone compared with the observational daily data by Gray \& Livingston. Left: comparison with the raw daily data; right: comparison between smoothed data, obtained by with a running mean over one year.

\section{Periodicity analysis}

\subsection{Line depths}

We study the periodicities of the different terms contributing to the line variations. In particular, we use the formalism developed by Lomb (1976) and Scargle (1982).

In order to avoid problems due to the biggest temporal gaps, we analyze only the period from 1978 to 1992.

All the periodograms are applied to the raw data, i.e. without smoothing operation. The power spectra relating to full-disk are shown in Fig. 7, while in Fig. 8 we report the power spectra of the center-disk data, comparing it with the "remainder" derived from the difference between the full-disk observational data and the AR contribution, reconstructed via models. The periodicities having a confidence greater than $99.9 \%$ are reported in Table 4. In this table we do not report the periodicities of less than $1 \mathrm{yr}$, but we may remark that all the data contain the solar rotation signal from 0.07 to $0.085 \mathrm{yr}$.

We note that all the data share a periodicity near to the cycle and a periodicity of about $2.8 \mathrm{yr}$. Moreover the full-disk data also have a periodicity of $1 \mathrm{yr}$, that is ascribable to the Earth's orbit, i.e. to variation of the magnetic structure size projected on the grating over the solar surface due to the orbital motion, and one of $1.4 \mathrm{yr}$.

The presence of the magnetic cycle periodicity in the diskcenter data is not obvious, because they should be free from magnetic elements; actually the center-disk data are averaged over 2 arcmin, i.e. over granulation and enhanced network, which could change with the cycle. In particular, the network affects the Fe I and Ti II lines much more than C I.

Summarizing, the lines seem to sense the effect of the magnetic cycle, even if with a different temporal response from each other, but are simultaneously affected by some other 

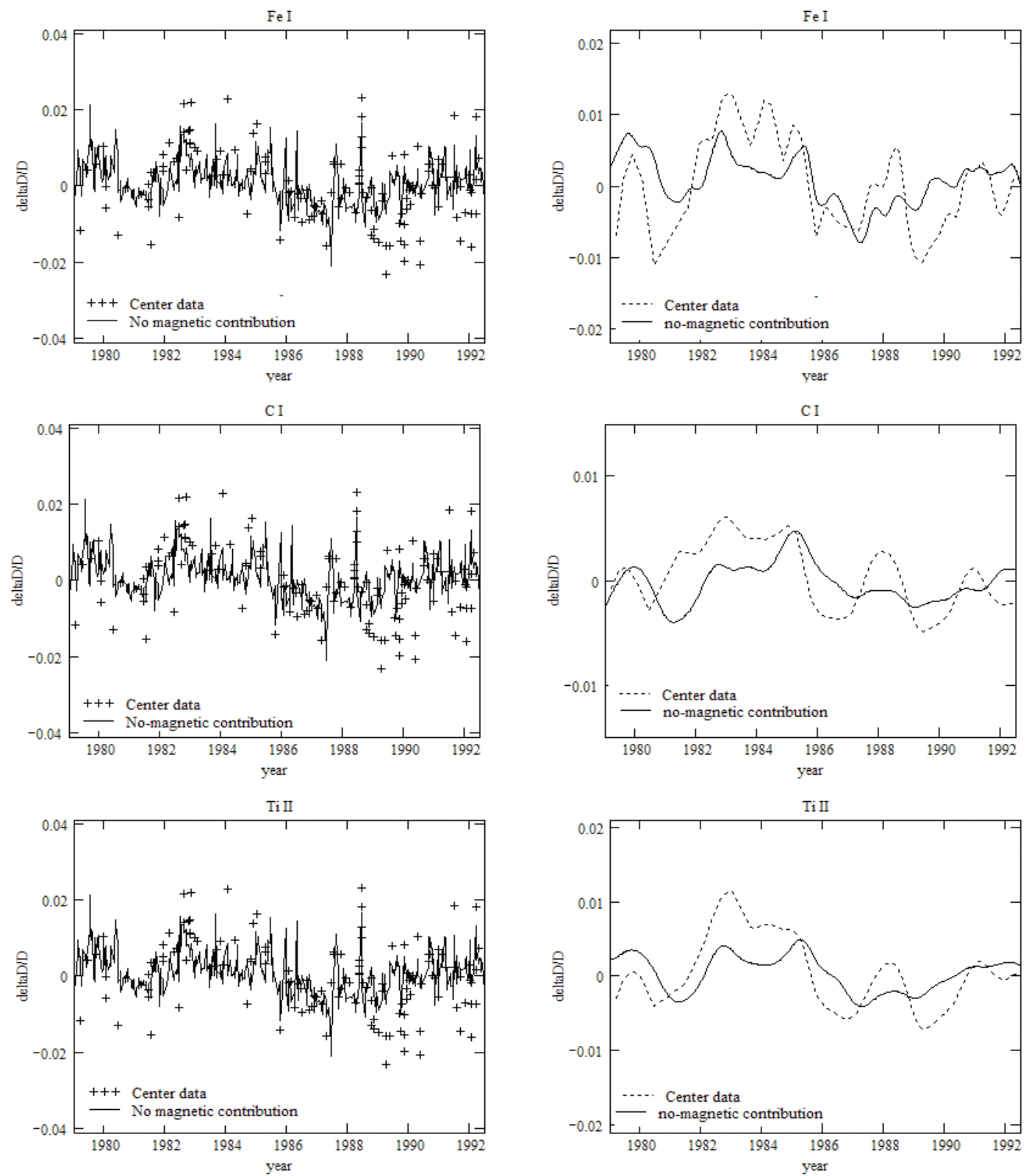

Fig. 5. Comparison of the variations of the line depth at center-disk of the three lines with the non-magnetic contribution, determined by the differences between the full-disk variations and the AR contribution. Left: comparison with the raw daily data; right: comparison with smoothed data, obtained by a running mean over 1 year.

phenomenon, with a period of $\sim 2.8$ yr. This could be a signal of a background contribution, which is, in principle, not strictly linked to magnetic activity.

In order to verify that the periodicities at $2.8 \mathrm{yr}$ are not mathematical harmonics of higher periods (e.g. the 11-yr cycle) we have repeated the analysis by splitting the sample, i.e. excluding the longest periodicities. The result is a substantial peak, slightly shifted towards $3 \mathrm{yr}$.

We can repeat this analysis separately also for the dataset covering the period 2000-2005, but there are sufficient data only for the full-disk measurements. The result remains that the $2.8 \mathrm{yr}$ periodicity is recovered.

\subsection{Solar activity indexes}

In order to understand whether the periodicities found in the photospheric lines are a "peculiarity" of this kind of data, or whether they are common to other data samples, we repeat the temporal analysis, via the Lomb periodogram, for some solar data such as facular and spot coverages and the green coronal emission line (Fe XIV $530.0 \mathrm{~nm}$ ). These are available at http://www.ngdc.noaa.gov/stp/SOLAR/ ftpsolarcorona.html/. By looking at periodicities between 1 and $5 \mathrm{yr}$, we obtain the spectra reported in Fig. 9.

The analysis of the power spectra shows that the periodicity around 2-3 year is present in all activity indexes.

In Table 4 we report the most important periodicities for each index, neglecting those shorter than $1 \mathrm{yr}$. As is evident, the analyzed activity indexes show more numerous periodicities than the line depth signal. Some of these could be cycle harmonics; in fact, if we repeat the analysis with the split samples, most disappear, but a peak at 2.5-2.7 yr persists in all of data.

Our results are perfectly compatible with those obtained by the analysis of Kane (2005) on similar solar indexes. 
Table 3. Line periodicities (in yr) obtained by power spectra of the two line measurement series (full and center-disk) and those corresponding to the difference between full-disk data and ARs contribution.

\begin{tabular}{ccc|ccc|ccc}
\hline \hline \multicolumn{4}{c}{ Full Disk (yr) } & \multicolumn{3}{c}{ Center Disk (yr) } & \multicolumn{3}{c}{ Residual (yr) } \\
\hline $\mathrm{Fe}$ & $\mathrm{C}$ & $\mathrm{Ti}$ & $\mathrm{Fe}$ & $\mathrm{C}$ & $\mathrm{Ti}$ & $\mathrm{Fe}$ & $\mathrm{C}$ & $\mathrm{Ti}$ \\
9.5 & 8.6 & 8.50 & 10.5 & - & 10.5 & 11.1 & 7.9 & 11.2 \\
- & - & - & - & - & & 6.2 & - & 6.2 \\
2.78 & 2.9 & 2.90 & 2.76 & 2.9 & 2.8 & 2.92 & 2.7 & 2.9 \\
- & 1.33 & 1.39 & - & - & - & - & - & 1.4 \\
- & 1.13 & - & - & & & & - & - \\
1.01 & 1.02 & 1.01 & - & - & - & - & 1.05 & - \\
\hline
\end{tabular}

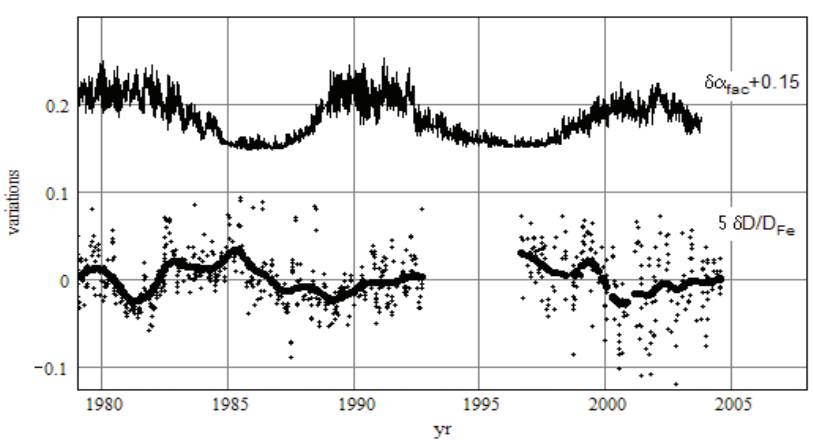

Fig. 6. Variations of the Fe line depth (bottom) and of the facular coverage $(u p)$. In order to plot the data on a unique graph, the first ones have been multiplied by 5 and the second ones have been shifted in the $y$ axis.

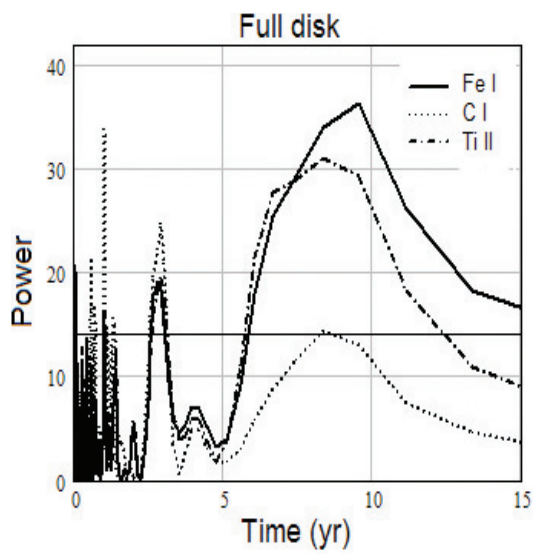

Fig. 7. Power spectra obtained by applying a Lomb periodogram to the observational line-depth data measured at full-disk data (Gray \& Livingston 1997a). The error confidence limit of $99.9 \%$ is reported.

We have tried to reconstruct the variations of line depths of three photospheric lines that were monitored for more than twenty years, both in full and at center-disk.

We have considered two possible contributions to their variations. The first one is a magnetic contribution, arising from the sensitivity of the lines to the active regions, and to their coverage variations along the cycle. By considering the variations due only to the active regions we are able to reproduce the TSI variations from 1978 to 2004, but not all the variations of the lines. In particular, neither the amplitude nor the phase seem well reproduced by AR contributions alone. This second effect has been denoted as a "non-magnetic contribution", where this appellation can contain any effect not strictly linked to faculae, spots and network. The difference between the observational line variations at full-disk with the evaluated AR contribution has been compared with the observational line variations at center-disk
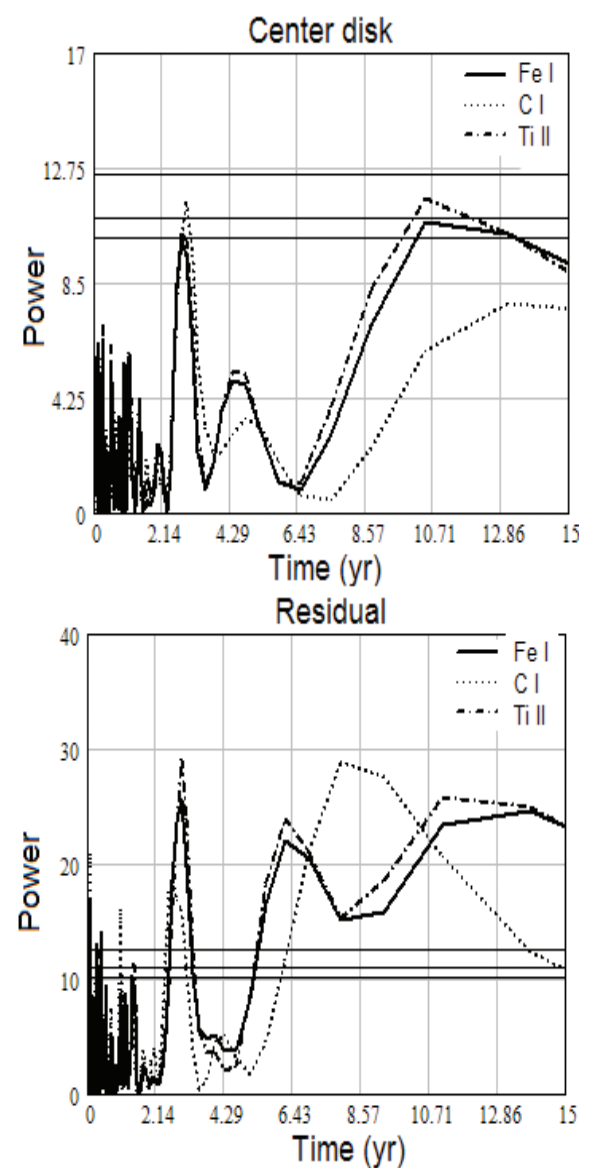

Fig. 8. Left: power spectra obtained by applying a Lomb periodogram to the observational line-depth data measured at center-disk data (Livingston 2003) of all three lines. Right: power spectra of the "remainder" deriving from the difference between the full-disk data and the AR contribution, reconstructed with the models. In both plots error confidence limits of $99.9 \%, 99.5 \%$ and $99 \%$ are reported, from the highest power value to the lowest one, respectively.

(theoretically not affected by AR presence). The two data series results again correlate.

We stress that the lines could be affected by multidimensional effects (corresponding both to granulation structure and to flux tubes) that cannot be included when we use 1-dimensional plane-parallel models and neglect the velocity fields and the corresponding line shifts and asymmetries. This should lead, when the disk integration is carried out, to an artificially smaller lineprofile.

A periodicity analysis (that takes into account the uneven sampling of the data) has highlighted the existence of a quasibiennial modulation (about $2.8 \mathrm{yr}$ ), shared by all the lines, both 

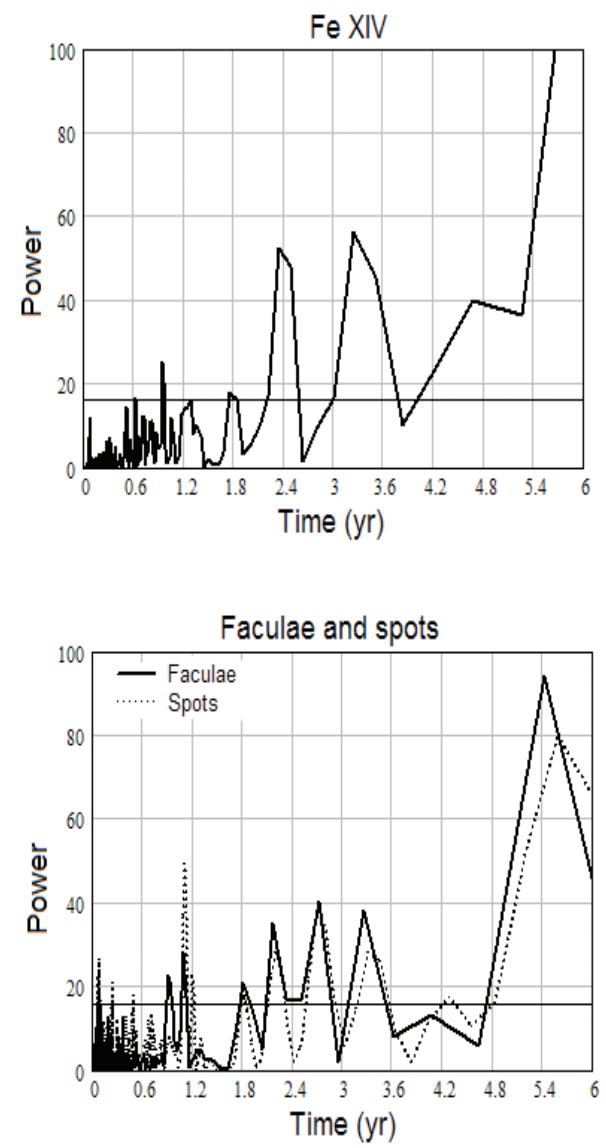

Fig. 9. Up: power spectra of Fe XIV $530.0 \mathrm{~nm}$ emission. Down: power spectra of facula and spot coverages. For both of graphs the time range is restricted to $1-5 \mathrm{yr}$ and a confidence level of $99.9 \%$ is found.

Table 4. Periodicities of different activity indexes analyzed via LombScargle periodogram.

\begin{tabular}{lll}
\hline \hline Faculae $(\mathrm{yr})$ & Spots $(\mathrm{yr})$ & Fe XIV $(\mathrm{yr})$ \\
\hline 10.8 & 10.4 & 9.5 \\
5.45 & 5.55 & - \\
- & 4.13 & 4.68 \\
3.25 & 3.20 & 3.25 \\
2.7 & 2.7 & 2.4 \\
2.17 & 2.2 & - \\
1.8 & 1.8 & 1.76 \\
- & 1.19 & - \\
\hline
\end{tabular}

at full and at center-disk, and by other activity indicators, such as facular and spot areas, and the coronal index.

The physical mechanism generating the quasi-biennial modulations is unknown. In this context, we can only note that the hypothesis of two dynamo sources (Benevolenskaya 1998) could explain our results.

The capability of reconstructing the TSI variations by imposing the AR area variations alone (and the impossibility to do the same thing with the line depths) is probably due to the lesser sensitivity of the total irradiance to several changes with respect to the line depths. For example, we known that it is possible to have the same total flux by synthesizing one dimensional atmospheric models having different parameters; in fact the flux depends on $T_{\text {eff }}$ alone, while line depth can depend on the gravity, on the abundance, and on the value of microturbulence.
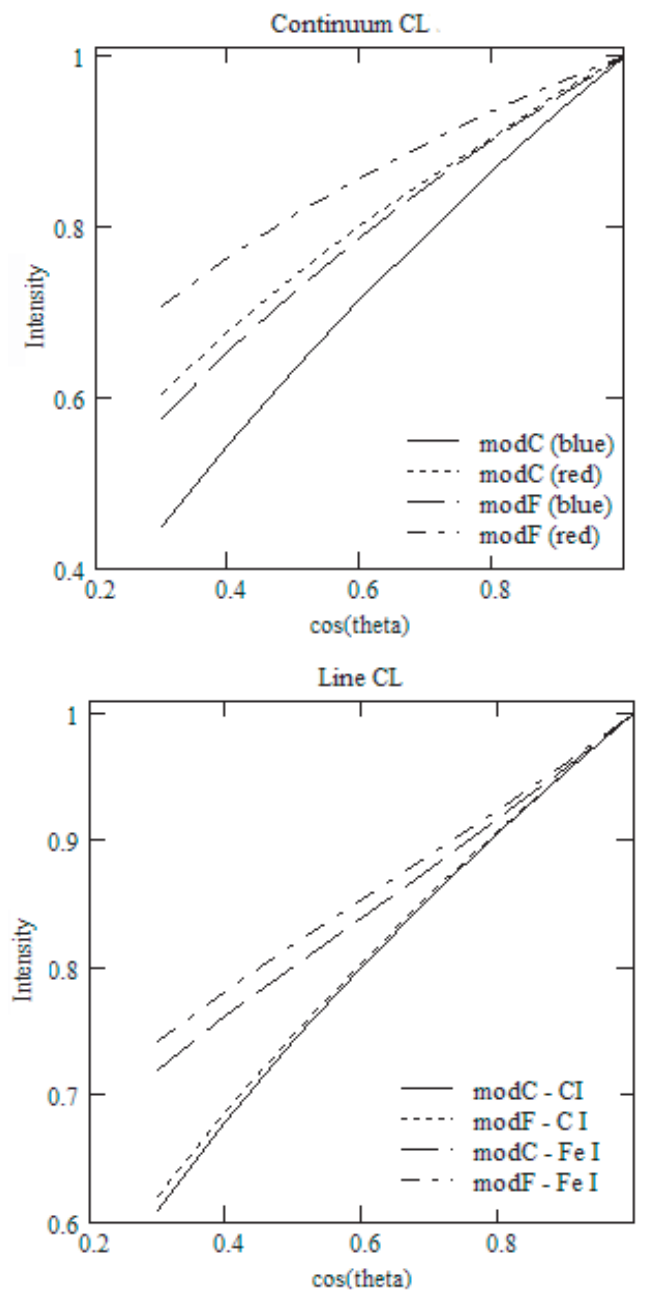

Fig. A.1. Up panel: center to limb trend of the continuum intensity in two different photometric bands (blue at $410.0 \mathrm{~nm}$ and red at $609.0 \mathrm{~nm}$ ) obtained by the modC (quiet sun) and modF (facular structure). Down panel: center to limb trend of the line core intensity of C I and Fe I obtained by the modC (quiet sun) and modF (facular structure).

Acknowledgements. We acknowledge unpublished data from the VIRGO Experiment on the cooperative ESA/NASA Mission SoHO.

The San Fernando Observatory is owned and operated by California State University Northridge, and its operations are funded by the University, NASA and NSF. The author thank G. Chapman and S. Walton for having provided spot and facula coverages for the Cycle 22 and 23.

The authors thank Roberto Piazzesi and Sabatino Sofia for useful comments.

Part of this work was supported by grants of CVS, sponsored by Regione Lazio.

\section{Appendix A: Estimate of error in neglecting $\mu$-dependence}

We consider a square facular zone of area A and centered at $\left(\theta_{0}, \phi_{0}\right)$. Calculating the total emission by weighing directly the fluxes, we have:

$\bar{F}=F_{\mathrm{c}}-A\left(F_{\mathrm{f}}-F_{\mathrm{c}}\right)$

where $F_{\mathrm{c}}$ and $F_{\mathrm{f}}$ are the fluxes of the model of the average sun (modC) and of the model of a facula (e.g. modF).

On the contrary, considering the intensities $\mu$-dependent, we would have:

$F=F_{\mathrm{c}}+\frac{1}{\pi} \int_{\phi_{1}}^{\phi_{2}} \int_{\theta_{1}}^{\theta_{2}}\left(I_{f}-I_{\mathrm{c}}\right) \sin \theta \cos \theta \mathrm{d} \phi \mathrm{d} \theta$ 

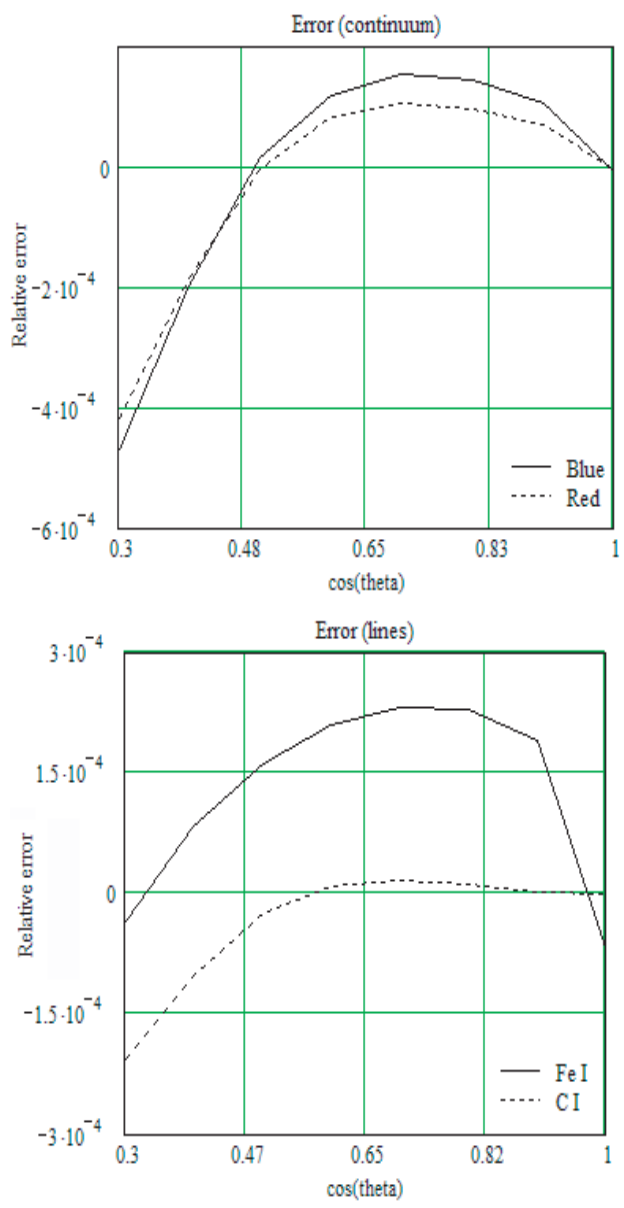

Fig. A.2. The error we commit by calculating the solar flux, using directly the model fluxes instead the intensity at the corresponding $\mu$. In the left panel the relative error for the continuum is reported; in the right panel the relative error for the line cores (for Fe and $\mathrm{C}$ ) is reported. All of this error corresponding to an active region area, considered as representative, $A=0.005$.

where

$\theta_{1,2}=\theta_{0} \pm \frac{\cos \phi_{0}+\sin \phi_{0}}{\cos \theta_{0}} \frac{\sqrt{A}}{2}$

and

$\phi_{1,2}=\phi_{0} \pm \frac{\cos \phi_{0}-\sin \phi_{0}}{\sin \theta_{0}} \frac{\sqrt{A}}{2}$.

Then, defining $\mu_{1,2} \equiv \cos \theta_{1,2}$, we have:

$F=F_{\mathrm{c}}+\frac{\sqrt{A}}{\pi} \frac{\cos \phi_{0}-\sin \phi_{0}}{\sin \theta_{0}} \int_{\mu_{2}}^{\mu_{1}}\left(I_{f}-I_{\mathrm{c}}\right) \mu \mathrm{d} \mu$.
By knowning the center to limb variations $I(\mu)$, calculated via FAL model spectral synthesis, both in the continuum and in the lines (Fig. A.1), we can estimate the error by using Eq. (A.1) instead of the more correct Eq. (A.5). As shown in Fig. A.2, the error is negligible (because quite smaller than intrinsic variations) both for the continuum and for the lines; moreover it changes in sign, i.e. is positive for large $\mu$ and negative for small $\mu$, so errors could, in some way, self compesate.

\section{References}

Baldwin, M. P., Gray, L. J., Dunkerton, T. J., et al. 2001, Rev. Geophys., 39, 179 Benevolenskaya, E. E. 1998, ApJ, 509, L49

Caccin, B., \& Penza, V. 2000, ESA, SP-463, 293

Caccin, B., Gomez, M. T., Marmolino, C., \& Severino, G. 1977, A\&A, 54, 227

Caccin, B., Penza, V., \& Gomez, M. T. 2002, A\&A, 386, 286

Chapman, G. A., Herzog, A. D., \& Lawrence, J. K. 1986, Nature, 319, 654

Ermolli, I., Centrone, M., \& Fofi, M. 2001, Mem. SAIT, 72, 673

Fligge, M., Solanki, S. K., \& Unruh, Y. C. 2000, A\&A, 353, 380

Fontenla, J., White, O. R., Fox, A. P., Avrett, E. H., \& Kurucz, R. L. 1999, ApJ, 518,480

Fontenla, J. M., Harder, J., Rottman, G., et al. 2004, ApJ, 605, L85

Frölich, C. 1994, in The Sun as a Variable Star, ed. J. M. Pap, C. Frölich, H. S. Hudson, \& S. K. Solanki, IAU Coll., 143, 28

Fröhlich, C. 2003, ESA SP-535 (Noordwijk: ESA Publications Division), 183

Gnevyshev, M. N. 1977, Sol. Phys., 51, 175

Gray, D. F., \& Livingston, W. C. 1997a, ApJ, 474, 802

Gray, D. F., \& Livingston, W. C. 1997b, ApJ, 484, 511

Gray, R. O. 1994, AJ, 107, 742

Kane, R. P. 2005, Sol. Phys., 227, 155

Krivova, N. A., Solanki, S. K., Fligge, M., \& Unruh, Y. C. 2003, A\&A, 399, L1 Kurucz, R. L. 1994, CD-ROM No. 19

Livingston, W., \& Wallace, L. 2003, Sol. Phys., 212, 227

Lomb, N. R. 1976, Ap\&SS, 39, 447

Lydon, T. J., Gunther, D. B., \& Sofia, S. 1996, ApJ, 456, L127

McIntyre, M. 1994, in the Solar Engine and Its Influence on Terrestrial Atmosphere and Climate, NATO ASI Ser. I 25, ed. E. Nesme-Ribes (BerlinHeidelberg: Springer-Verlag), 293

Penza, V., Caccin, B., Ermolli, I., Centrone, M., \& Gomez, M. T. 2003, ESA SP-535 (Noordwijk: ESA Publications Division), 299

Penza, V. 2003, Ph.D. Thesis

Penza, V., Caccin, B., Ermolli, I., \& Centrone, M. 2004a, A\&A, 413, 1115

Penza, V., Caccin, B., \& Del Moro, D. 2004b, A\&A, 427, 345

Penza, V., Caccin, B., \& Del Moro, D. 2004c, in Multi-Wavelength Investigations of Solar Activity, Proc. IAU Symp., 223, 137

Roudier, Th. 2003, ESA Publ. Ser., ed. J. Arnaud, \& N. Meunier, 9, 191

Scargle, J. D. 1982, ApJ, 263, 835

Sofia, S., Oster, L., \& Schatten, K. 1982, Sol. Phys., 80, 87

Tritschler, A., \& Schmidt, W. 2002, A\&A, 388, 1048

Unruh, Y. C., Solanki, S. K., \& Fligge, M. 1999, A\&A, 345, 635

Unruh, Y. C., Solanki, S. K., \& Fligge, M. 2000, Space Sci. Rev., 94, 145

Walton, S. R., Preminger, D. G., \& Chapman, G. A. 2003a, ApJ, 590, 1088

Walton, S. R., Preminger, D. G., \& Chapman, G. A. 2003b, Sol. Phys., 213, 301

Willson, R. C., \& Hudson, H. S. 1991, Nature, 351, 42 